\title{
PREVALENCE OF OVERWEIGHT, OBESITY AND RISK OF INJURY IN CZECH SCHOOL
} CHILDREN

\author{
J. Vignerova ${ }^{1}$, S. Janoušek ${ }^{2}$, Z. Roth ${ }^{2}$, P. Sedlak ${ }^{3}$, V. Faierajzlová ${ }^{1}$, Z. Zvadová ${ }^{1}$ \\ ${ }^{I}$ Dept. of Health Promotion and Child and Adolescent Health, ${ }^{2}$ Dept. of Toxicology and Veterinary Services, \\ National Institute of Public Health, ${ }^{3}$ Dept. of Anthropology and Genetics, Faculty of Science, Charles \\ University, Praha, Czech Republic
}

Background and aims: Czech Republic is one of the countries with increasing prevalence of child overweight and obesity. Current epidemiological data highlight a significant increase in the child injury rate. The aim of this presentation is to determine the prevalence of overweight and obesity and point out the relationship between overweight and obesity, and the increased risk of injury incidences.

Methods: Data on 2839 children, ages 7, 9, 11 and 13 years, were selected from a large 2009 study on the incidence rate of injuries in school children. Using IOTF criteria, each child was clasified as either normal, overweight or obese. Fractures were found the most frequent injuries (34\%), followed by contusions (22\%). Relative risk of increased incidence of fractures and contusions in children with overweight or obesity was calculated using the method $\chi^{2}$.

Results: The prevalence of overweight and obesity increased in the studied age groups between 2001 and 2009 (overweight: boys $18.9 \%$, girls $15.1 \%$; obese: boys $5.1 \%$, girls $2.9 \%$ vs overweight: boys $13.4 \%$, girls $13.1 \%$; obese: boys $3.3 \%$, girls $2.8 \%$ ). The results confirmed an increased risk of fractures in children with overweight and obesity, boys and girls alike (boys: $R R=1.55$, CI 1.17-2.05; girls: $R R=1.37$, CI 0.99-1.81). The lowest RR values were found in the group of 11-year olds.

Conclusions: In relation to the problem of overweight and obesity, attention should be paid also to the prevention of incidents in school children.

The research is supported by grant of Ministry of Health no. NS 9208-4 / 2008. 